\title{
Using various point wise and multi-group cross section libraries in MORET criticality calculations
}

\author{
Joachim Miss $^{1}$, Olivier Jacquet ${ }^{2}$, Franck Bernard ${ }^{1}$, Isabelle Duhamel ${ }^{1}$, Stéphane Evo ${ }^{1}$, and Marc Leclere ${ }^{1}$ \\ 1 Institut de Radioprotection et de Sûreté Nucléaire (IRSN), Fontenay-aux-Roses, France \\ 2 Independent consultant, Salignac, France
}

\begin{abstract}
Most modern criticality safety Monte Carlo codes have reached a good level of achievement concerning the implemented simulation algorithms. Discrepancies observed on final results (for example performed by different teams with different codes and data) are mainly due to the used nuclear data. Therefore, knowing and controlling the way of processing nuclear data libraries used by these codes is nowadays crucial. Recent MORET code (developed by IRSN) improvements have focused on the development of a prototypic continuous energy version. Indeed, so far, the cross sections used by the MORET code are read from external multi-group libraries. It has been then decided to plan new developments in MORET 5 to control the whole criticality calculation process, from the reading of nuclear data to the Monte Carlo simulation itself. The new features related to this new version will allow performing criticality calculations either with a multi-group or a continuous energy treatment. The goal of this paper is to present the main topics needed to ensure proper development of a continuous energy version of MORET, and the results concerning the use of various multi-group cross sections libraries in MORET.
\end{abstract}

\section{Introduction}

The MORET [1] multi-group code is a Monte Carlo criticality code commonly used in France and developed by IRSN. It solves the transport equation for neutral particles in threedimensional systems, calculates both effective multiplication factor (k-effective), reaction rates in the different volumes of the geometry and the leakage of neutrons out of the system. So far, the cross sections used by the MORET code could only be taken from external multigroup libraries resulting from preliminary calculations performed by default with the APOLLO2 cell code (processed from the JEF evaluation). It has now been decided to plan new developments in MORET 5 to control the whole process of criticality calculation, from the reading of nuclear data to the Monte Carlo simulation. The main new features related to this new version are:

- the transparent use of various standard multi-group cross sections libraries (from the SCALE package or DRAGON code), allowing to study more precisely the discrepancies observed between results performed with different criticality codes combinations;

- criticality calculations will now be able to be performed either with a multi-group or a continuous energy treatment;

- the implementation of the Woodcock tracking method should improve computation time for non-homogenized configurations with many small volumes.

The aim of this paper is to focus on the first results concerning the use of various multi-group cross sections libraries with MORET 5. Thus, we briefly describe the developments of the prototypic continuous energy version of MORET 5. More information will be available in the papers presented at ICNC 2007 [2] and M\&C 2007 [3].

\section{New MORET 5 capabilities}

\subsection{Why developing a point wise version?}

Multi-group approach may sometimes give incorrect results, in particular for configurations with very steep neutron flux heterogeneities, and when the self-shielding models inherent to the multi-group treatment are not well adapted. The continuous energy approach is therefore essential in those cases. The main issues in development of a continuous energy version of MORET are:

- the control of the tools allowing the processing of continuous energy cross section libraries for MORET from any basic evaluation (JEF, ENDF...);

- the adaptation of the tracking routines in MORET to the new point wise description (memory requirements, anisotropy and thermal treatment...);

- the study of solutions to reduce simulation time of reference calculations (including the Woodcock tracking strategy, and the use of an universal energy grid or a very fine multi-group mesh).

As IRSN carries out research, analysis and work within the fields of nuclear safety in France, it is important to estimate the bias induced by the use of different cross sections libraries. Such reflections are currently investigated by IRSN for criticality safety analysis. For instance, the experimental validation database of the APOLLO2-MORET 4 standard route of the CRISTAL package is composed of more than 1800 benchmarks models, mainly issued from the ICSBEP Handbook, covering a broad variety of configurations in terms of fissile medium, moderator, reflector and neutron spectrum. Multi-sets of calculations were performed on this large validation database using group-wise (172 and 281 groups) nuclear data libraries based respectively on JEF2.2, JEFF3.1 and ENDF/B.VI evaluation files (see fig. 1). 


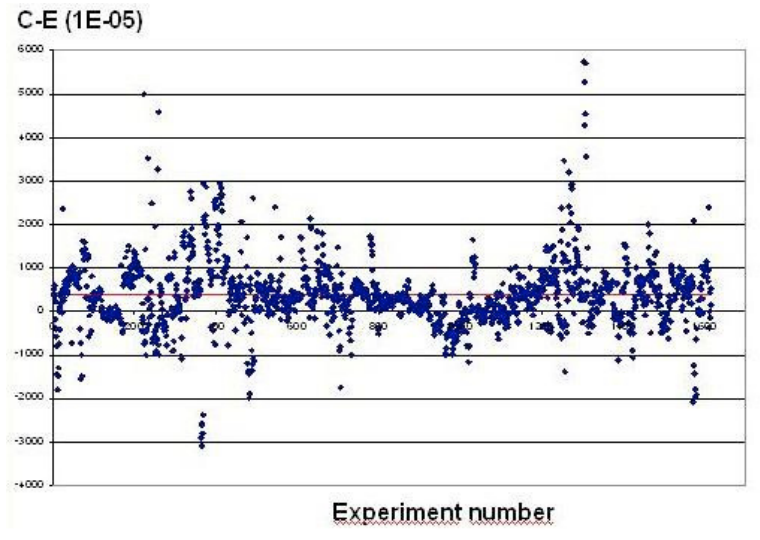

Fig. 1. Discrepancies between calculations and experimental (C-E) results.

In order to control these nuclear data issues, it is obviously crucial to understand the way of producing cross sections libraries. As for now the development of cross sections processing tool is not planed, the production of nuclear data libraries is performed using the common continuous energy processing code, NJOY [4]. Moreover, because the MCNP cross sections data are extensively used in the world, the ACE format was selected.

By now, MORET 5 is able to read and store the information of ACE cross sections files, and allows generating new ACE cross sections files with an universal energy grid; indeed using universal energy grid for all the isotopes handled in a Monte Carlo calculation is expected to merge some benefits for both continuous and multi-group energy modeling.

Many developments are now devoted to the porting of the tracking routines available in the multi-group route to the point wise version. Simultaneously, several approaches of variance reduction are implemented like Woodcock tracking [5]. Some of these developments are described below.

\subsection{Improvement of the MORET multi-group route}

This study aims at adding to the MORET multi-group route the capability to perform reference calculations using a very fine energy mesh of several thousand groups, and to benefit from the efficiency of a multi-group treatment in terms of simulation time. It is well known that for point wise calculations, the higher the number of output tallies (with cross section multipliers), the longer the calculation will take, which is not the case for multi-group runs.

The basic idea is that, with a sufficiently fine multi-group mesh, the cross sections are almost independent of the flux. The use of a universal 11276 group mesh could allow to treat correctly the non resolved range and the upper part of the resolved range [6].

Our first tests showed we could use in MORET about 8000 groups with the non-optimized transfer matrices to treat the Rowland benchmark. As a proof of concept, we modify the MORET code in order produce fictitious very fine multigroup cross sections from standard XMAS (172 groups) cross sections transmitted to MORET by standard lattices codes, in order to study the evolution of the memory used during the Monte Carlo simulation. MORET reads these cross sections and divides each group artificially by an integer factor, and rebuilds the new finer multi-group cross sections by dividing the cross sections values for each group by this factor.

\subsection{Improvement of the MORET continuous energy route}

A point wise cross section consists of tabulated energy cross section values so that it is necessary to find the proper energy interval and to apply an appropriate interpolation treatment to deduce the value of the cross sections at an energy $E$ within the energy interval.

It is necessary to run this step each time when new cross section values are needed during the Monte Carlo simulation, namely for every interaction, for sampling the length of the probable track before the next collision and for scoring.

For instance the sampling of the probable track length requires the computation of the total macroscopic cross section of the material in a cell, and as a consequence the computation of the total microscopic cross section of each isotope of this material. The computation time then increases with the number of isotopes in the material, because (in the general case) each isotope has its own energy grid so that the search of the proper energy interval has to be repeated for each isotope. As a result, the calculation time within a cell is roughly proportional to the number of tracks in the cell multiplied by the number of isotopes. This point is detailed in the paper presented at M\&C 2007 [3].

The performance of Monte Carlo codes could therefore be improved if each isotope of a material used the same energy grid so that the calculation time becomes independent of the number of isotopes in each material. The first simple idea to build a universal energy grid is to merge the energy grids of each isotope. However these grids use very few common points because they are produced independently from each other by the RECONR module of NJOY. As a consequence, the size of the universal grid would never cease of growing with the number of isotopes to handle in the Monte Carlo calculation. As the memory size is constrained, this approach is not satisfactory.

The main issue remains to make the Monte Carlo code capable of merging entire nuclides into a single grid and keeping just the number of points necessary to represent each nuclide with a given accuracy. The algorithm used in RECONR to produce a unique grid to represent all the neutron reactions of a nuclide with a linear-linear approximation could be adapted as a first step.

\section{Using various standard multi-group cross sections libraries with MORET 5}

MORET 5 multi-group Monte Carlo calculations are possible with direct use of various multi-group cross section sets generated by different routes: APOLLO [7], DRAGON [8] and SCALE system [9]. Each of these codes or system 
relies on independently processed data libraries. So, intercomparison of these results is relevant for verification of the global consistence of multi-group data. This way of making is particularly interesting to highlight problems in the process of libraries.

For DRAGON-MORET calculations, we built our own multi-group libraries by using, and modifying PyNjoy [10] entries to perform group-to-group scattering matrix at the order 5, because freely available libraries with DRAGON, dedicated to reactor calculations, were limited to first order.

An extensive validation is in progress based on the experimental validation database of MORET, including more than 1800 benchmarks models. Some criticality benchmarks tested and performed with MORET are extracted from this database (see fig. 2), coming from experiments chosen among the International Criticality Safety Benchmark Evaluation Project (ICSBEP).

Results point out that the choice of data and their processes are the main issues. As a consequence, this approach is suitable to validate the methods for generating the multi-group scattering cross section matrices.

Results highlight the following points:

- consistent results in general with the use of JEF2.2 multigroup libraries,

- possible under-estimation of diffusions in water and moderated fissile media probably due to our multi-group cross sections processing with PyNjoy for DRAGON4-MORET 5 (JEF3.1) calculations,

- need for checking SCALE cross sections reading by MORET to point if the discrepancies come either from the way of processing our self-shielded and homogenized multi-group cross sections, or from a misunderstanding of the SCALE generated cross sections format.

More precise analysis is still in progress using the calculations for the whole database with the various multi-group cross section sets.

\section{Conclusions}

This paper summarizes the current status of the developments of the MORET 5 criticality code, outlining research and innovations dedicated to develop a new multi-group and continuous energy version. This report provides also information about possible systems using MORET when combined with different cell codes. The first very encouraging results were not performed to show the performance of the system used, but mainly point out that the choice of data and their processing are of great importance.

A large part of the last MORET developments has been concerned with implementing and validating the reading of

口 case 1: D4-M5 (Jef2.2)

ncase 2: D4-M5 (Jef3.1) In case 3: D4-M5 (endfb6) a case 4: A2-M5 (Jef2.2 : P1-P5) ase $5:$ : A2-M5 (Jef2.2 : P5) Ucase 6: A2-M15 (Jef3.1) (M5: MORET5; D4: DRAGON4; = case 7: A2-M5 (endfb6) A2: APOLLO2; S5: SCALE5) $\square$ case 8: S5-M5(endfb6)
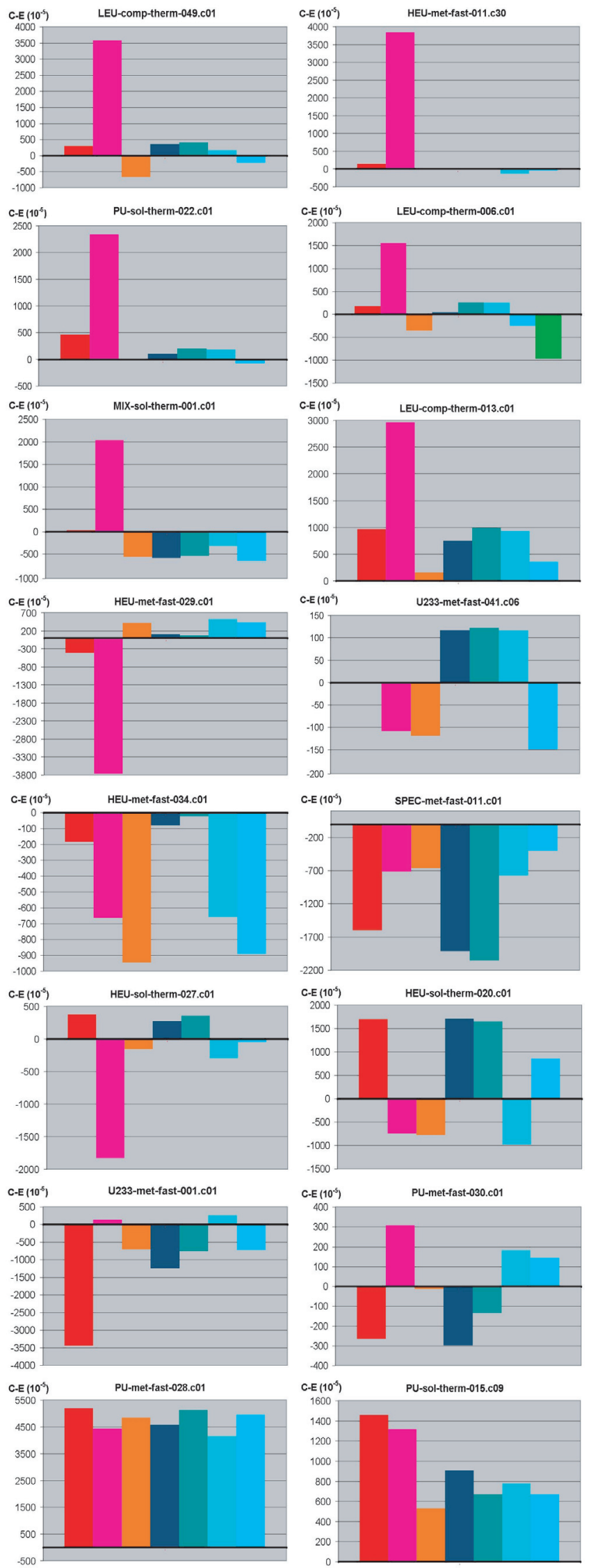

Fig. 2. Extract of the experimental validation database performed with MORET 5 using different multi-group cross section sets. Discrepancies between calculations and experimental results. 
ACER cross section data files. This work successfully led to the reading of every MCNP cross sections with MORET. Main developments now focus on the tracking Routines, and so to adapt the different methods available in the multi-group route to the specificities of the point wise version. For now, the development of our own cross sections processing tool is not planed, but it will undoubtedly be necessary in futher steps. The NJOY understanding manual mentioned the following issue:

"The NJOY Nuclear Data Processing System is used to convert evaluated nuclear data in ENDF format into forms useful for applications. As a bridge between physics and engineering, it is best used by people with some knowledge of things like nuclear reaction theory, resonance theory, or scattering theory on one side, and some knowledge of things like particle transport codes, reactor core calculations, or radiation medicine on the other".

So, using tools like "black boxes" to process cross sections files is not sufficient and relevant work need to efficiently understand and use the different NJOY modules.

To conclude, some MORET 5 features are studied to find various means to reduce the computing times inherent to point wise treatment of the energy. Such "satellite" projects of the point wise version of MORET 5 code, concern the three items summarized below.

- The use of the Woodcock tracking algorithms to lower computation time both in the multi-group and point wise energy approach.

- The use of a very fine energy mesh of several thousand groups for the multi-group approach, without needing preliminary calculations performed with a cell code (necessary to take into account self-shielding and homogenization).

- The use of an universal energy grid (the same for all isotopes and media) for a continuous energy treatment.
Regular feedbacks of the MORET user's community held considerably the developers to improve the new implementations. Thanks to them.

\section{References}

1. J. Miss, O. Jacquet, L. Heulers, F. Fernex, Y. Richet, in Proceedings of M\&C2005, Avignon, France, The MORET 4.B Monte Carlo code. New features to treat complex criticality systems.

2. J. Miss, O. Jacquet, F. Bernard, B. Forestier, M. Leclere, in Proceedings of ICNC2007, St Petersbourg, Russia, Massive evolution in the MORET Monte Carlo code. A new multi-groupcontinuous energy version.

3. J. Miss, O. Jacquet, B. Forestier, in Proceedings of $M \& C 2007$, Monterey USA, Feasibility of using a universal point wise energy grid or a very fine multi-group energy grid in MORET5 criticality calculations.

4. R. Mac Farlane, Nucl. Data Sheets (to be published), NJOY nuclear data processing code, version 2006.

5. E.R. Woodcock, T. Murphy, P.J. Hemmings, T.C. Longworth, ANL 7050 (1965), Techniques used in the GEM code for Monte Carlo neutronics calculations in reactors and other systems of complex geometry.

6. S. Mengelle, Note CEA-N-2800, APOLLO2: Reference calculation using a fine energy mesh.

7. S. Loubière et al., in Proceedings of Int. Conf. Mathematical and computation, reactor physics and environnemental analysis in nuclear applications, Madrid, 1999, APOLLO2, twelve years later.

8. G. Marleau, A. Hébert, R. Roy, Technical Report, IGE-294, A user guide for DRAGON version 4.

9. SCALE: A Modular Code System for Performing Standardized Computer Analyses for Licensing Evaluations, ORNL/TM2005/39, Version 5.1, Vols. I-III, November 2006.

10. A. Hébert, R. Karthikeyan, Interfacing NJOY with advanced lattice codes. 